\title{
PRECISION OF THE HALL QUANTIZATION IN NATURALLY OCCURRING TWO-DIMENSIONAL SYSTEM - HgCdMnTe BICRYSTALS
}

\author{
G. Grabecki ${ }^{a}$, A. Wittlin ${ }^{a, b}$, T. Dietr ${ }^{a}$ P.A.A. Teunissen ${ }^{b}$, \\ S.A.J. WIEGERS ${ }^{b}$ AND J.A.A.J. PERENBOOM ${ }^{b}$ \\ ${ }^{a}$ Institute of Physics, Polish Academy of Sciences \\ Al. Lotników 32/46, 02-668 Warszawa, Poland \\ ${ }^{a}$ High Field Magnet Laboratory and Research Institute for Materials \\ University of Nijmegen, Toernooiveld, 6525 ED Nijmegen, The Netherlands
}

New magnetoresistance measurements on naturally occurring inversion layers adjacent to grain boundaries in narrow gap diluted magnetic semiconductor $\mathrm{HgCdMnTe}_{\mathrm{g}}\left(E_{\mathrm{g}} \leq 200 \mathrm{meV}\right)$ have been performed. The magnetic fields up to $20 \mathrm{~T}$ simultaneously with millikelvine temperatures have been applied. Possible experimental factors affecting the quantum Hall effect in our system are discussed in detail.

PACS numbers: 72.20.My, 72.80.Ey, 73.40.Lq

Present theoretical models regard quantum Hall effect (QHE) as a consequence of fundamental properties of 2D systems, see for innstance [2]. Most of them suggest that QHE is independent of such details of two-dimensional.(2D) systems as: 2D potential well shape, sample geometry, material of the sample, degree of disorder in the sample etc. This indicates that the effect should occur even in strongly imperfect 2D systems. However, in real experiments, there are additional factors limiting the possibility of the observation of QHE [4]:

1. The disorder in the $2 \mathrm{D}$ sample should not be too strong, because otherwise the mixing of Landau levels will preclude formation of minima in the density of states.

2. Precise measurements of the QHE plateau height require bypass resistance, short-circuiting the Hall resistance to be appropriately high.

The presence of QHE in HgCdMnTe grain boundaries (GB) was demonstrated in our previous work [3]. However, even at millikelvine temperatures the precision of the Hall quantization was smaller than that reported for III-V heterostructures [1], and longitudinal resistance $\left(R_{\mathrm{L}}\right)$ minima exhibited values greater than 0 . Unambiguously, in that case the QHE was affected by the above described obscuring effects. The main goal of the present work is an experimental study of this problem. 


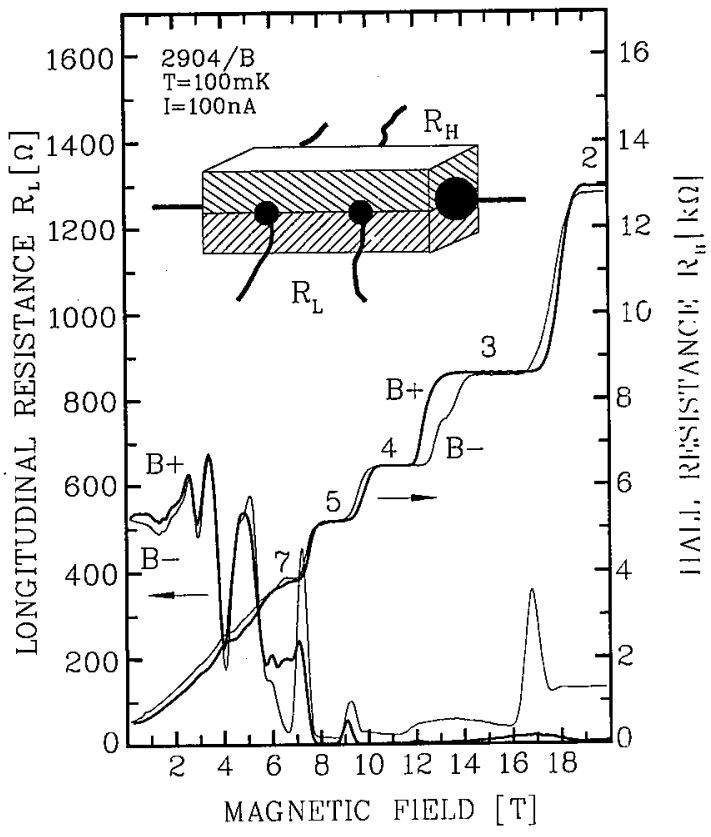

Fig. 1. Diagonal and Hall resistances of $\mathrm{HgCdMnTe} \mathrm{GB}$ with $2 \mathrm{D}$ electron concentration $N_{S}=1.1 \times 10^{12} \mathrm{~cm}^{-2}$ taken at $100 \mathrm{mK}$ for both directions of the magnetic field $(B+$ thick lines, $B-$ thin ones). The inset shows the sample scheme.

Typical six-contact geometry sample with single GB is presented in the inset of Fig. 1. In order to carry out magnetotransport measurements we used dilution refrigerator installed in Bitter coil capable of producing magnetic fields up to $20 \mathrm{~T}$. Experimentally obtained $R_{\mathrm{L}}$ and $R_{\mathrm{H}}$ magnetic field dependencies at $T=100 \mathrm{mK}$ for one $\mathrm{HgCdMnTe}$ GB are presented in Fig. 1 for both magnetic field directions $B+$ and $B-$. It is clearly visible that for both magnetic field directions the $R_{\mathrm{H}}$ plateaux and $R_{\mathrm{L}}$ minima (up to $i=7$ ) occur at the same magnetic field regions. This is an evidence that our sample is well homogeneous [5]. Estimated 2D electron density gradient between measuring probes does not exceed $10 \%$.

As one can see from Fig. 1, the $R_{\mathrm{L}}$ minima show nonzero values $\mathrm{R}_{x x}^{\min }>0$. The magnetoresistance oscillations are superimposed on the monotonically increasing background (Fig. 2). The background resistance is proportional to the square of the magnetic field, $R_{x x}^{\min }=C B^{2}$. The proportionality factors $C$ are different for different contact pairs (Fig. 2(a) and (b)) and for the two magnetic field directions (see Fig. 1). It was noted by von Klitzing and Ebert [4] that such an effect resulted from the presence of electrical bypass between measuring contact probes. From experimentally obtained coefficients $C$ one can estimate values of bypass resistance $R_{X}$. For the sample presented in Fig. $1(2904 / \mathrm{B})$ we have $R_{X}=1.5 \mathrm{M} \Omega$ for $B-$ and $R_{X}=25 \mathrm{M} \Omega$ for $B+$. Temperature independence of the $R_{X}$ for $T<0.5 \mathrm{~K}$ indicates that the bypass resistances have a metallic character. 


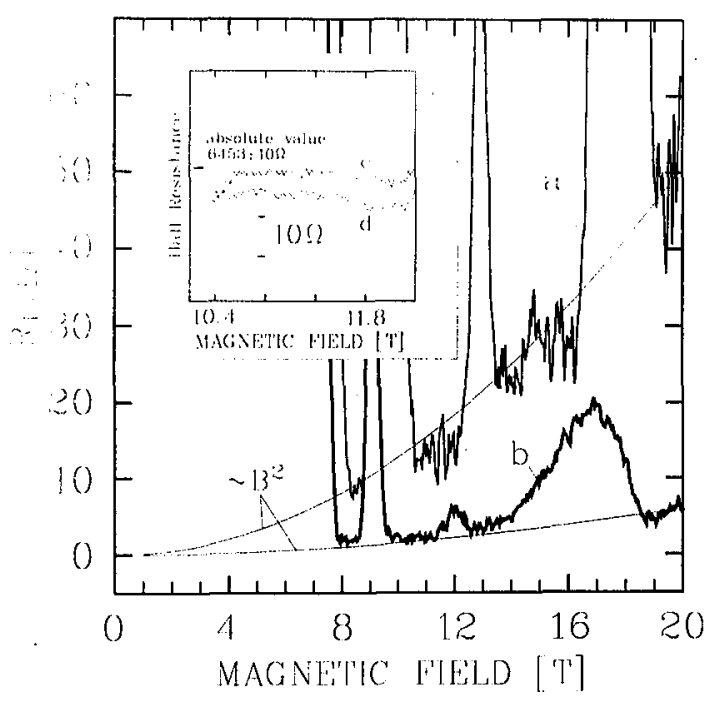

Fig. 2. Magnified parts of the $R_{\mathrm{L}}(\mathrm{B})$ measured for both contact pairs (lines a and $\mathrm{b}$ ) along the sample sides. The inset: magnified Hall plateaux for quantum index $i=4$, measured between both contact pairs across the sample presented in Fig. 1 (curves a and $b$ ).

The values of $R_{X}$ are considerably smaller than those suggested by resistance measurements on the reference bulk material $(R \gg 20 \mathrm{M} \Omega)$. This fact may be understood if one assume that the bulk resistance in the GB close proximity (say, at the distance less than several $\mu \mathrm{m}$ ) has smaller resistivity. One natural possibility is the presence of increased amount of crystallographic defects, like dislocations or $\mathrm{Hg}$ precipitates, in this region. Such defects may lead to formation of conducting chains, short-circuiting different parts of the inversion layer.

These defects may be characterized by their specific, random spatial distribution along the CB plane. This fact may explain the asymmetry of the $R_{X}$ values with respect to the magnetic field direction. It is well known that under. QHE conditions, the electric current density across our 2D sample is very inhomogeneously distributed [2]. If the region with a high local current density occur in the GB part surrounded by low resistive bulk, the short-circuiting will be very effective. Analogically, the high density current paths may be positioned in regions neighbouring high resistive bulk material and the apparent bypass resistance will be very high. Because current distribution in inhomogeneous samples depend on the magnetic field direction, it implies a similar dependence of the measured bypass resistance $R_{X}$.

The presence of finite bypass resistances (and thus, non-vanishing $R_{x x}^{\min }$ ) is the main limitation on the precise measurements of the Hall quantization. It has been well established that the deviation from the exact quantized value:

$$
\Delta R_{x y}=\alpha R_{x x}^{\min },
$$


where the dimensionless factor $\alpha$ is of unity order. Figure 2 (inset) illustrates accuracy of the Hall quantization in our sample for the plateau corresponding to the quantum index $i=4$. The inhomogeneity of the bypassing effect implies different deviations from quantized values, observed for different contact pairs and field polarities. Using standard laboratory equipment we are able to establish the absolute resistance value with accuracy of about $0.1 \%$. The plateau slope has been established to be as small as $5 \times 10^{-5}$ of the total Hall resistance on the distance of $1 \mathrm{~T}$. We thus conclude that QHE is not affected by the presence of magnetic ions up to this accuracy level.

\section{References}

[1] M.E. Cage, B.F. Field, R.F. Dziuba, S.M. Girvin, A.C. Gossard, D.C. Tsui, Phys. Rev. B 30, 2286 (1984); H.L. Stormer, D.C. Tsui, A.C. Gossard, Surf. Sci. 113, 32 (1982).

[2] H. Aoki, Rep. Prog. Phys. 50, 655 (1987).

[3] G. Grabecki, T. Suski, T. Dietl, T. Skośkiewicz, M. Gliński, in: High Magnetic Fields in Semiconductor Physics, Ed. G. Landwehr, Springer-Verlag, Berlin 1984, p. 127.

[4] K. von Klitzing, G. Ebert, in: Two-Dimensional Systems, Heterostructures and Superlattices, Eds. G. Bauer, F. Kuchar, H. Heinrich, Springer-Verlag, Berlin 1984, p. 242.

[5] D.A. Syphers, P.J. Stiles, Phys, Rev. B 32, 6620 (1985). 